\title{
Element Analysis by EDX for Life Science: Light Elements and Bio-Mineralization.
}

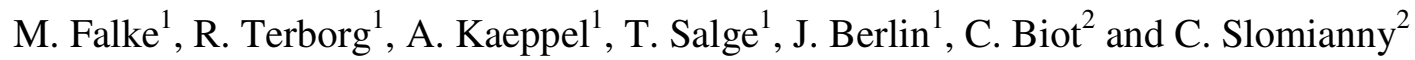 \\ ${ }^{1 .}$ Bruker Nano GmbH, Schwarzschildstr. 12, 12489 Berlin, Germany \\ ${ }^{2 .}$ Laboratory of Cell Physiology, University of Lille, France
}

Beam sensitivity of biological samples represents a challenge for electron microscopy and respective spectroscopy methods. Apart from different low dose techniques, one way to reduce beam damage effects and enable stable spectroscopic signals is the use of accelerating voltages below 80keV. High brightness sources combined with aberration correction can achieve the resolution of nanometer sized objects and even single atoms at low voltages in TEM [1]. This paper contributes examples including very standard SEM and TEM set ups to elaborate on the question, to which extent and under which conditions the limited radiation yield from small carbon based structures can be used for element analysis by energy dispersive spectroscopy (EDX).

Silicon drift detector (SDD) systems have become state of the art technology for EDX. One main advantage is the extremely high efficiency. This means, that due to light element windows and fast pulse processing at low electronic noise, most of the incoming photons contribute to the available signal. For single channel EDX in SEM and TEM the electron beam current, solid angle, take-off angle and exposure time can be optimized to investigate the light element composition and bio-mineralization of particular samples. Multiple SDD setups provide even higher efficiency and larger collection angles for the X-ray analysis. Shadowing effects in element distribution maps are minimized and the measurement time is further reduced. The robustness of SDDs allows their integration into the microscope pole piece for further geometrical optimization [2]. SDD systems provide high energy resolution also in the low EDX energy range. Elements such as $\mathrm{B}, \mathrm{C}, \mathrm{N}, \mathrm{O}, \mathrm{P}$ or S and heavier elements causing L- and M-lines in that energy range can be distinguished routinely. Elements used for labeling in life science, e.g. Ag and $\mathrm{Au}$, even as nm sized particles, can be imaged. Additionally, modern data processing provides a range of advanced analysis options.

Figures 1-2 show a TEM analysis of human erythrocytes, infected with Plasmodium falciparum - the malaria parasite. Osmium was used for fixation. Malaria parasites invade erythrocytes in the host bloodstream and induce crucial changes to these cells during their reproduction cycle. Nutrients for their growth are obtained by degrading hemoglobin. The parasites mature, divide and at complete maturity burst out of the blood cell. The EDX mappings show the element distribution in one infected and the surrounding healthy erythrocytes. The sample was treated with a derivate of chloroquine, which leads to the incorporation of chlorine into the erythrocyte membranes and the parasites cytoplasm, as visible in the quantified Cl-map. The Fe enrichment in the food vacuole of the parasite can be studied.

These data suggest that with the latest technological developments in microscope and detector instrumentation, new options to study biological samples in SEM and TEM become available. EDX is ideally suited for identifying and quantifying many elements at once, including those used for fixation, staining and labeling and also light elements. For the utilization of EDX in life science and the diagnosis of diseases, respective sample preparation techniques need to be adapted. Is staining still necessary to answer certain questions? Could additional elements, easily distinguishable by EDX, be used for labeling different objects of interest in a cell or protein? 
References:

[1] T.C. Lovejoy et al., accepted by Appl. Phys. Lett. (2012).

[2] S. von Harrach et al., Microsc. Microanal. 15, Suppl. 2(2009) 208

and Schlossmacher et al., Microscopy Today 18(4) (2010) 14-20.
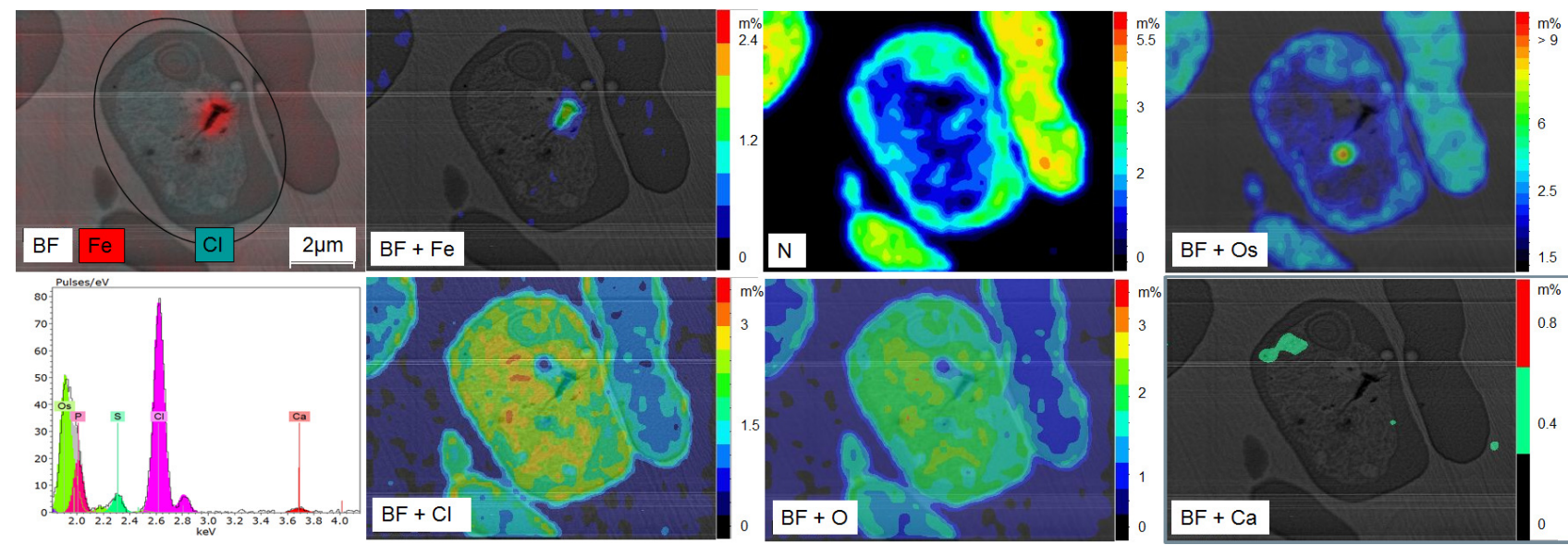

Figure 1. Quantified element distributions of $\mathrm{Fe}, \mathrm{N}$, Os (fixation), $\mathrm{Cl}, \mathrm{O}$ and $\mathrm{Ca}$ in a human blood sample infected with Plasmodium (apart from N, all overlaid to the bright field image). Note the Cldistribution showing the successful chloroquine treatment and the Fe content of the Plasmodium parasite's food vacuole. Small amounts of Ca were found, which may be due to a preparation effect or contamination. The spectrum peak deconvolution (lower left) demonstrates how elements were identified and quantified. Thin black line: experimental spectrum, colors and grey for the sum: deconvolution result after quantification.
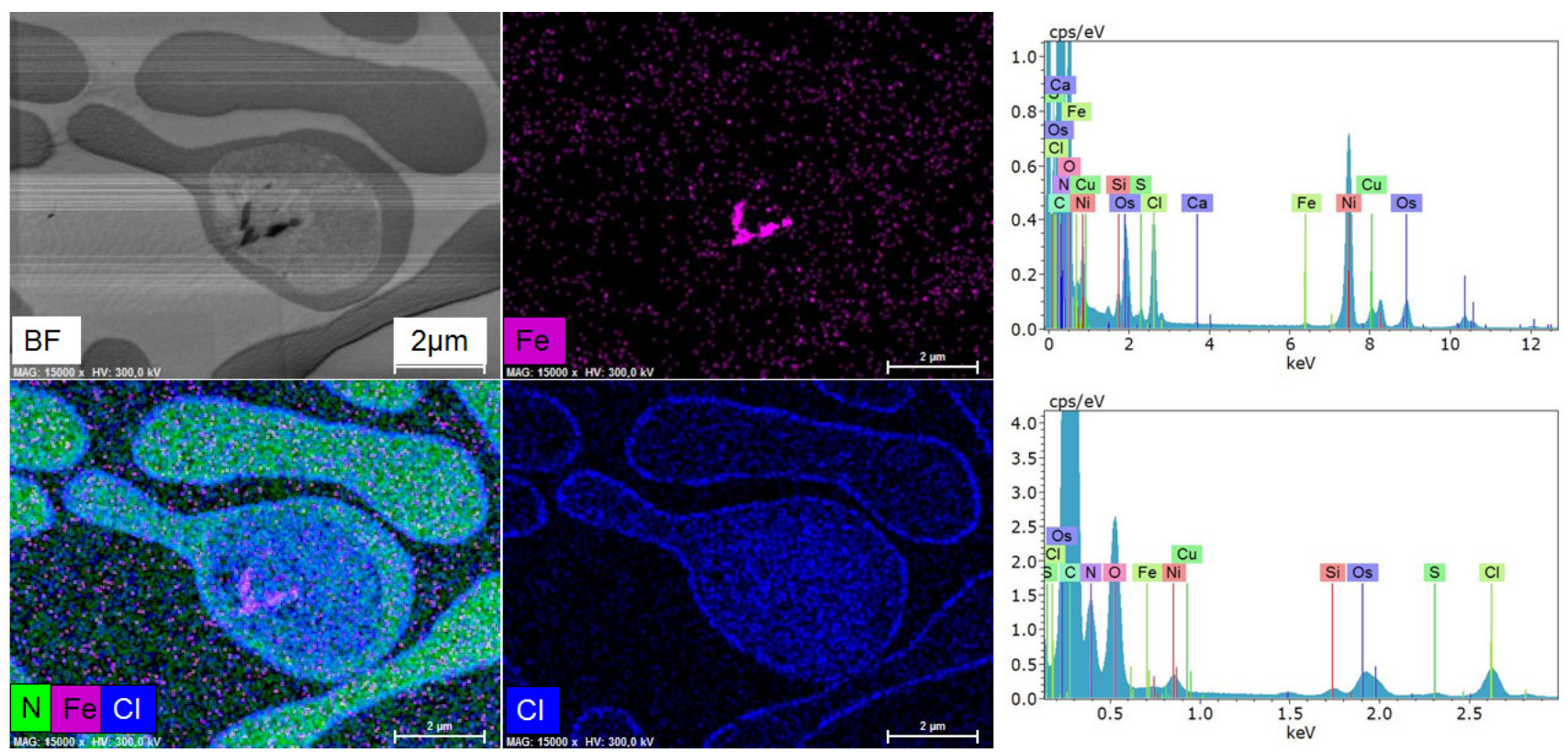

Figure 2. Another example of Plasmodium infected erythrocytes on a Ni-grid, investigated using TEM EDX: Here just qualitative element maps and the respective EDX spectrum overview are shown. 